\title{
Efecto antioxidante y hepatoprotector del Petroselinum sativum (perejil) en ratas, con intoxicación hepática inducida por paracetamol *
}

\author{
Luzmila Troncoso ${ }^{1}$, Emilio Guija ${ }^{2}$
}

Resumen

Palabras clave

\begin{abstract}
Objetivo: Determinar el efecto antioxidante y hepatoprotector del perejil (Petroselinum sativum) en ratas con intoxicación hepática inducida por paracetamol. Lugar: Centro de Investigación de Bioquímica y Nutrición - "Laboratorio de Bioquímica Clínica y Nutricional "Leonidas Delgado Butrón” "Emilio Guija Poma” - Universidad Nacional Mayor de San Marcos. Lima, Perú. Diseño: Estudio analítico, transversal, prospectivo y cuasi-experimental. Material: Ratas albinas Holtzman machos adultas. Métodos: Se utilizó 40 ratas de 2 meses de edad, con pesos entre 280 y $320 \mathrm{~g}$, distribuidas aleatoriamente en cuatro grupos de 10 animales cada uno. Todos los grupos recibieron la misma dieta y agua ad libitum, además de los respectivos tratamientos, los cuales fueron administrados por vía oral diariamente, durante 5 días: paracetamol (administrado en una dosis de $200 \mathrm{mg} / \mathrm{kg}$ de peso corporal) para inducir la intoxicación hepática y, al mismo tiempo, un hepatoprotector, ya fuera farmacológico (fármaco hepatoprotector (FHP): Purinor ${ }^{\circledR}$ ) o natural (perejil); además, un grupo de paracetamol solo y otro de control. Al término del período experimental, los animales fueron sacrificados. En suero sanguíneo se determinó aspartato aminotransferasa (AST), alanina aminotransferasa (ALT), gamma glutamil transferasa (GGT), grupos sulfhidrilo, proteínas totales y albúmina sérica; y en el homogenizado citosólico de hígado, fracción posmitocondrial, se determinó superóxido dismutasa, catalasa, glucosa-6-fosfato deshidrogenasa, grupos sulfidrilo, especies reactivas al ácido tiobarbitúrico (TBARS) o radicales libres y proteínas. Además, se realizó el estudio histopatológico del hígado, para identificar signos de necrosis y signos de regeneración posnecrótica. Principales medidas de resultados: Efecto antioxidante y hepatoprotector del perejil. Resultados: El perejil mostró un mejor efecto hepatoprotector que el FHP, frente a la acción nociva del paracetamol, evaluado por AST, ALT y GGT. La glucosa-6-fosfato deshidrogenasa ( $p<0,05, p=0,01$, prueba Kruskal-Wallis) y las TBARS ( $p<0,001, p=0,000$, prueba Kruskal-Wallis) permitieron mostrar que existió diferencia estadisticamente significativa entre todos los grupos. Histopatológicamente, se observó signos de necrosis severa con la administración solo de paracetamol y en el grupo al que se administró adicionalmente FHP, no encontrándose mayores cambios en el grupo tratado además con perejil. Conclusiones: El perejil ejerce un mayor efecto antioxidante y hepatoprotector que el FHP.
\end{abstract}

Perejil; medicamentos hepatoprotectores; acetaminofeno; antioxidantes; toxicidad.
Petroselinum sativum (perejil) antioxidant and hepatoprotective effects in rats with paracetamolinduced hepatic intoxication

Abstract

Objective: To determine the antioxidant and hepatoprotective effect of parsley (Petroselinum sativum) in rats with

* Trabajo de Tesis para optar el Grado Académico de Doctor en Medicina. Facultad de Medicina, UNMSM. Lima, Perú.

1 Centro de Investigación de Bioquímica y Nutrición. Facultad de Medicina, Universidad Nacional Mayor de San Marcos. Lima, Perú.

2 Profesor Emérito del Centro de Investigación de Bioquímica y Nutrición. Facultad de Medicina, UNMSM. Lima, Perú. paracetamol-induced hepatic intoxication. Setting: Leonidas Delgado Butron - Emilio Guija Poma Clinical and Nutritional Biochemistry Laboratory, Biochemistry and Nutrition Research Center, Universidad Nacional Mayor de San Marcos, Lima, Peru. Design: Analytical, transverse, prospective and quasi-experimental study; only 'post' with quasi-control group design. Biologic materials: Adult male Holtzman albino rats. Methods: We utilized forty 2 months-old adult rats weighing 280 to 320 $g$ distributed at random in four groups 10 animals each. All groups received the same ad libitum diet and water along with respective treatments administered orally daily 
during 5 days: paracetamol was administered $200 \mathrm{mg} / \mathrm{kg}$ pc) to induce hepatic intoxication and concurrently a pharmacologic (hepatoprotective drug (HPD): Purinor ${ }^{\circledR}$ ) or natural (parsley) hepatoprotector; another group was treated with paracetamol only and there was a control group. The animals were sacrificed at the end of the experimental period. We determined in serum aspartate aminotransferase (AST), alanine aminotransferase (ALT), gamma glutamyl transferase (GGT), sulphidril group, total proteins and serum albumin; and in liver postmitochondrial fraction cytosolic homogenates we determined superoxide dismutase, catalase, glucose-6-phosphate deydrogenase, sulphidril group, thiobarbituric acid reactive species (TBARS) or free radicals and proteins. Besides, histology study of the liver was done to identify both signs of necrosis and postnecrotic regeneration. Main outcome measures: Parsley's antioxidant and hepatoprotective effects. Results: Parsley showed a better hepatoprotective effect than HPD against paracetamol's nocive effect, as evaluated by AST, ALT and GGT serum levels. Determination of glucose-6-phosphate dehydrogenase ( $p<0,05, p=0,01$, Kruskal-Wallis test) and TBARS ( $p<0,001, p=0,000$; Kruskal-Wallis test) in liver cytosolic showed the existence of statistically significant difference among all groups. On histology we observed signs of severe necrosis after administration of paracetamol alone and in the group with additional HPD, with no further changes in the group treated additionally with parsley. Conclusions: Parsley exerts a greater antioxidant and hepatoprotective effect than HPD.

Key words: Petroselinum; hepatoprotector drugs; acetaminophen; antioxidants; toxicity.

\section{INTRODUCCIÓN}

El paracetamol ha sido utilizado en diversos estudios, habiéndose mostrado en ratas y ratones que tiene la propiedad de inducir lipoperoxidación y daño irreversible en el hepatocito, probablemente causados por radicales libres $\left({ }^{1}\right)$.

Los radicales libres de oxígeno son compuestos químicos caracterizados por poseer uno o más electrones desapareados. Algunos de ellos son extremadamente reactivos, como el radical hidroxilo $\left({ }^{\circ} \mathrm{OH}\right)$, otros menos reactivos como el radical superóxido $\left(\mathrm{O}_{2}^{-}\right)$y el peróxido de hidrógeno $\left(\mathrm{H}_{2} \mathrm{O}_{2}\right)$, que por definición no es considerado un radical libre de oxígeno, pero es potencialmente capaz de generar fácilmente ${ }^{\circ} \mathrm{OH}$. Los radicales libres pueden formarse intracelularmente en los peroxisomas, en la cadena transportadora de electrones, durante la fagocitosis, la autooxidación o como consecuencia de la interacción de metales de transición, como el hierro o cobre con ascorbato o peróxido de hidrógeno $\left.{ }^{(2-5}\right)$. Ciertos compuestos químicos ingeridos en la dieta, diversas sustancias tóxicas (humo del cigarrillo), radiaciones electromagnéticas, ozono y algunos medicamentos (paracetamol) pueden ejercer su acción nociva en el organismo a través de la generación de radicales libres, los que pueden dañar carbohidratos, lípidos, proteínas y ácidos nucleicos y, como consecuencia de ello, dañar seriamente las membranas celulares $\left({ }^{2,3,5}\right)$.

Cada vez se presenta más evidencias que demuestran la participación de los radicales libres en la patogénesis de ciertas enfermedades, como cáncer, artritis reumatoide, arteriosclerosis, diabetes mellitus, catarata senil, enfermedad de Alzheimer, Kwashiorkor y procesos como el de la inflamación $y$ el envejecimiento $\left({ }^{2,3,5-9}\right)$.

El organismo dispone de sistemas de defensa antioxidante que actúan impidiendo la formación de radicales libres, bloqueando su propagación o interaccionando directamente con ellos. Integran este sistema la superóxido dismutasa, catalasa, glutation peroxidasa, glutation reductasa, ácido úrico, proteínas, glucosa, glutation, grupos sulfidrilos (-SH), entre otros. También, es posible que se ingiera con la dieta sustancias naturales o de origen sintético con capacidad antioxidante, como flavonoides, polifenoles, $\beta$-caroteno, vitamina $E$, vitamina $C$, dimetilsulfóxido y otros más $\left({ }^{2,10}\right)$.

El uso de las plantas medicinales con fines curativos es una práctica que se ha venido utilizando desde tiempo inmemorial. Éstas tienen la mayoría de los antioxidantes, principalmente polifenoles y flavonoides, los cuales poseen gran actividad antioxidante. Se ha estudiado las propiedades antiinflamatorias y antioxidantes del tomillo (Thymi herba) $\left({ }^{11}\right)$. Los extractos acuosos de Coptis chinensis, Paeonia suffruticosa, Prunilla vulgaris y Senecio scandens mostraron una alta capacidad antioxidante frente a sistemas generadores de $\mathrm{O}_{2}$ y ${ }^{\bullet} \mathrm{OH}$, inhibiendo la lipoperoxidación en riñón y homogenizado de cerebro de ratas $\left({ }^{12}\right)$. 
La ingesta de antioxidantes presente en los alimentos es un factor protector de la salud importante. En estudios anteriores hemos mostrado in vitro que el perejil presenta evidente efecto antioxidante $\left({ }^{13-16}\right)$, al igual que las fresas y mucho mayor que otros alimentos, como naranja, brócoli $\left({ }^{17}\right)$, ajos, cebolla, limón $\left({ }^{18,19}\right)$, pimiento $\left({ }^{20}\right)$, cebolla china $\left({ }^{21}\right)$, betarraga $\left({ }^{22}\right)$, hojas de coca y anís $\left({ }^{17}\right)$ evaluados frente a sistemas ascorbato/Cu(II), generador de ${ }^{\bullet} \mathrm{OH}$ y phenazina metosulfato (PMS)/nitro blue tetrazolio (NBT)/nicotin adenin dinucleótido reducido $(\mathrm{NADH})$, generador de $\mathrm{O}_{2}{ }^{--}$.

El perejil (Petroselinum sativum) es una umbelífera bianual que contiene apiína y flavonoides, compuestos que le confieren acción diurética y antioxidante; aceite esencial rico en apiol y miristicina, que le otorga propiedades emenagogas (estimula la menstruación) y vasodilatadoras. Contiene vitaminas A, C y E, fósforo, hierro, calcio y azufre. Las mujeres embarazadas deben evitar consumir grandes cantidades de perejil, por tener cierto efecto oxitócico (contrae el útero), que podría predisponer al aborto $\left({ }^{23-25}\right)$.

Todos estos aspectos nos conducen a suponer que los alimentos que posean poder antioxidante, como el perejil (Petroselinum sativum), debido a su composición química, puedan ser utilizados para prevenir la hepatotoxicidad generada por algunas drogas, como el paracetamol, en cuyo mecanismo patogénico intervienen precisamente por un lado la producción de radicales libres y por otro la alteración del sistema antioxidante a nivel hepático.

El objetivo de esta investigación fue determinar el efecto antioxidante y hepatoprotector del perejil (Petroselinum sativum) en ratas con intoxicación hepática inducida por paracetamol.

\section{MÉTODOS}

Se realizó un estudio tipo analítico, transversal, prospectivo y cuasi-experimental. Se utilizó 40 ratas albinas Holtzman machos adultas, de 2 meses de edad, cuyos pesos estaban comprendidos entre 280 y 320 g, distribuidas de una manera aleatoria en cuatro grupos de 10 animales cada uno. Se colocó las ratas en jaulas individuales, en un ambiente de temperatura constante, con ciclos alternados de $12 \mathrm{~h}$ de luz y $12 \mathrm{~h}$ de oscuridad. Una semana antes de iniciar propiamente el experimento, las ratas solo recibieron una dieta normocalórica, normoproteica y agua ad libitum. Posteriormente, todos los grupos siguieron recibiendo la misma dieta y agua ad libitum, además de los respectivos tratamientos. Se administró los tratamientos por vía oral diariamente, durante 5 días, y estaban constituidos por paracetamol (administrado en una dosis de $200 \mathrm{mg} / \mathrm{kg}$ de peso corporal) para inducir la intoxicación hepática $\mathrm{y}$, al mismo tiempo, por un hepatoprotector, ya fuera farmacológico (FHP) o natural (perejil [Petroselinum sativum]).

Los tratamientos administrados a cada grupo fueron los siguientes: grupos control, a) al grupo control propiamente dicho se le administró $1 \mathrm{~mL}$ de agua destilada; b) el grupo paracetamol (grupo control de la inducción del daño) recibió solo paracetamol, en una dosis de $200 \mathrm{mg} / \mathrm{kg}$ de peso corporal. Los grupos experimentales: a) fármaco hepatoprotector (FHP) Purinor ${ }^{\varpi}$, recibió paracetamol y, además, se le administró una dosis de FHP de $50 \mathrm{mg} / \mathrm{kg}$ de peso corporal; b) el grupo perejil (Petroselinum sativum) recibió paracetamol y, además, extracto acuoso de perejil fresco, en una cantidad de $150 \mathrm{mg} / \mathrm{kg}$ de peso corporal.

Al término del período experimental, los animales, previo ayuno de 14 horas, fueron anestesiados por inhalación de cloroformo en una campana de vidrio. La sangre se extrajo por punción cardiaca y luego los animales fueron sacrificados por decapitación. Posteriormente, se separó el hígado, el que fue perfundido con una solución isotónica de $\mathrm{NaCl}$ y se colocó en una placa Petri sobre hielo.

El extracto de perejil fresco (Petroselinum sativum) se preparó licuando las hojas al $25 \%$ en agua bidestilada, luego se filtró a través de gasa y finalmente se centrifugó a $2500 \mathrm{rpm}$ durante 30 minutos. El sobrenadante obtenido fue administrado a los animales de experimentación, en una dosis de $150 \mathrm{mg} / \mathrm{kg}$ de peso corporal. Se centrifugó la sangre a $1500 \mathrm{rpm}$ en una centrífuga clínica, durante 20 minutos, y se separó el suero para realizar diversas determinaciones. Se pesó 5 g de hígado, 
se prefundió con $\mathrm{NaCl} 9 \%$ y se homogenizó con una solución $0,25 \mathrm{M}$ de sacarosa, tampón Tris- $\mathrm{HCl}$ $0,2 \mathrm{M} \mathrm{pH} \mathrm{7,4,} \mathrm{en} \mathrm{una} \mathrm{relación} \mathrm{de} \mathrm{1:5,} \mathrm{utilizando}$ un Potter-Elvehjem provisto de un pistilo de teflón. Luego, se centrifugó en una centrífuga refrigerada Sorvall RC-2B, a 10000 g, durante 45 minutos, a cuyo término se descartó el precipitado y se reservó el sobrenadante. Todo este proceso se realizó a una temperatura no mayor de $4^{\circ} \mathrm{C}$, en la cual también se mantuvo el sobrenadante que correspondía a la fracción posmitocondrial, hasta realizar las determinaciones bioquímicas correspondientes.

Para el estudio histopatológico hepático, se practicó cortes de $0,5 \times 1,0 \mathrm{~cm}$ de espesor, que fueron fijados en formol neutro al $10 \%$, los que fueron seccionados para inclusión en cortes de 2 $\mathrm{mm}$ de espesor; posteriormente, se efectuó cortes con micrótomo, en un espesor de $3 \mu$, para luego ser coloreados con HE (hematoxilina-eosina) y revisados con microscopio óptico, para la identificación de signos de necrosis en el parénquima y regeneración posnecrótica.

Se utilizó los siguientes reactivos: ácido 5,5'ditiobis (2-nitrobenzoico) (DTNB), 2-desoxirribosa, Tris, ácido cacodílico, pirogalol y ácido tiobarbitúrico, kit para la determinación de glucosa-6-fosfato deshidrogenasa (Sigma Chemical Company); ácido tricloroacético, fosfato de potasio, ácido clorhídrico, cloruro de sodio, hidróxido de sodio, formaldehído al 35\%, hematoxilina y eosina (Merck Darmstadt); vitamina C (ácido ascórbico), fosfato dihidrógeno de potasio, peróxido de hidrógeno al $30 \%$ (Riedel-de Haën); kits para transaminasas 200 (AST, ALT), $\gamma$-G-test cinética, Proti 2 (proteínas totales y albúmina) (Wiener lab); paracetamol (acetam, gotas) (Laboratorios COFANA); FHP (combinación de sustancias activas como orotato de potasio, xantina, adenina, fructosa difosfato de magnesio, sorbitol, tiamina clorhidrato, riboflavina-5-fosfato sódico, nicotinamida, pantenol, piridoxina clorhidrato, cianocobalamina) - Purinor $^{\oplus}$, suspensión oral (Laboratorio Farmacéutico Peruano - Germano S.A.); vitamina A (retinol palmitato) - Epiteliol ${ }^{\varpi}$, suspensión bebible (Medifarma Laboratorios); vitamina E (D- $\alpha$ tocoferil acetato) - Vitesol E 400 ${ }^{\circledast}$, cápsula líquida (UNIMED del Perú S.A.). Todas las soluciones fueron preparadas con agua bidestilada en vidrio neutro.
Se realizó las siguientes determinaciones en suero sanguíneo de las ratas: aspartato aminotransferasa (AST), según Reitman S y Frankel F $\left({ }^{26}\right)$, alanina aminotransferasa (ALT), según Reitman $S$ y Frankel F ${ }^{26}$ ), gamma glutamil transferasa (GGT), según Szasz G $\left({ }^{27}\right)$, grupos sulfidrilo, por el método de Ellman $\left({ }^{28}\right)$, proteínas totales con el método de Biuret $\left({ }^{29}\right)$, albúmina sérica con el método de verde de bromocresol $\left({ }^{29}\right)$. En sobrenadante hepático, se estudió superóxido dismutasa, según Del Maestro $\left({ }^{30}\right)$, catalasa, según Aebi $\left({ }^{31}\right)$, glucosa-6-fosfato deshidrogenasa, según Deutsch J ${ }^{(32}$ ), grupos sulfidrilo, por el método de Ellman $\left({ }^{28}\right)$, determinación de sustancias reactivas al ácido tiobarbitúrico (TBARS), según Ney K, Colley K y Pizzo S ${ }^{(33}$ ), proteínas con el método de Biuret $\left({ }^{29}\right)$.

La muestra total fue seleccionada por conveniencia según el peso corporal de las ratas y fue distribuida aleatoriamente en cuatro grupos de 10 animales cada uno. Luego de la ejecución del diseño experimental, los datos fueron ordenados y analizados, aplicándosele primero la prueba de normalidad, para seleccionar las pruebas de hipótesis estadísticas adecuadas. Al no tener la muestra una distribución normal, se aplicó la prueba Kruskal-Wallis, para comparar medias de más de dos grupos, y la prueba Mann-Whitney, para comparar medias de dos grupos. Se utilizó el valor $\mathrm{p}<0,05$ para la consideración de diferencia estadísticamente significativa. Para el análisis estadístico, se aplicó el paquete estadístico SPSS v.12.

\section{RESULTADOS}

La evaluación del efecto antioxidante y hepatoprotector de los diferentes tratamientos, tanto farmacológico (FHP) como natural o alimento medicinal (perejil), frente a la intoxicación hepática inducida por paracetamol en ratas dio los siguientes resultados:

El peso de las ratas al iniciar el experimento estuvo comprendido entre 280 y 320 g y terminó con un peso corporal final entre 320 y $340 \mathrm{~g}$. El peso corporal final y el peso absoluto del hígado no mostraron diferencia estadísticamente significativa entre los grupos, al igual que el peso relativo del hígado con respecto al peso corporal final (Tabla 1). 
Tabla 1. Pesose de ratas según tratamiento.

\begin{tabular}{lcccc}
\hline Tratamiento & \multicolumn{2}{c}{ Peso corporal } & & \multicolumn{2}{c}{ Peso del hígado } \\
\cline { 2 - 2 } \cline { 5 - 5 } & g & & g & \% p.c. \\
\hline Control $^{\mathrm{a}}$ & $339 \pm 17,5$ & & $13,7 \pm 4,1$ & 4,0 \\
Paracetamol $^{\mathrm{b}}$ & $329 \pm 54,0$ & & $11,7 \pm 2,0$ & 3,6 \\
FHP $^{\mathrm{c}}$ & $321 \pm 50,2$ & & $11,2 \pm 2,1$ & 3,5 \\
Perejil $^{\mathrm{d}}$ & $339 \pm 9,5$ & & $11,9 \pm 1,7$ & 3,5 \\
\hline
\end{tabular}

${ }^{a}$ Sin tratamiento. ${ }^{b}$ Tratamiento: paracetamol

${ }^{c}$ Tratamiento: paracetamol y Purinor ${ }^{\circledR}$ (FHP)

${ }^{d}$ Tratamiento: paracetamol y Petroselinum sativum (perejil)

${ }^{e}$ Media $\pm S D$; NS: No significativo; p.c.: peso corporal

En este estudio, con relación a los niveles séricos del aspartato aminotransferasa, no hubo diferencia estadísticamente significativa entre todos los grupos a un $p<0,05$, pero se pudo observar que la administración oral de paracetamol produjo mayores niveles (66 U/L) que el grupo control (56 $\mathrm{U} / \mathrm{L})$, presentando los menores niveles el grupo con perejil (45 U/L). En cambio, al comparar el grupo de perejil con el grupo de paracetamol, sí se observó una diferencia estadísticamente significativa ( $p<0,10$, prueba Mann-Whitney) (Tabla 2). En lo referente a los niveles séricos de actividad de la alanina aminotransferasa, tampoco pudimos observar diferencia estadísticamente significativa entre todos los grupos, a un $p<0,05$; sin embargo, sí existió diferencia al comparar el grupo de perejil (21 U/L) ( $p=0,03$, prueba Mann-Whitney) con respecto al grupo de paracetamol (24 U/L) (Tabla 2).

Tabla 2. Transaminasas ${ }^{\mathrm{e}}$ en suero sanguíneo de ratas según tratamiento.

\begin{tabular}{|c|c|c|c|}
\hline Tratamiento & $\begin{array}{c}\mathrm{ASTf}^{(\mathrm{NS})} \\
\mathrm{U} / \mathrm{L}\end{array}$ & $\begin{array}{c}\mathrm{ALT}^{\mathrm{g}} \\
\mathrm{U} / \mathrm{L}\end{array}$ & $\begin{array}{l}\mathrm{GGT}^{\mathrm{h}} \\
\mathrm{U} / \mathrm{L}\end{array}$ \\
\hline Control $^{\mathrm{a}}$ & $56 \pm 21,3$ & $23 \pm 4,9$ & $0,04 \pm 0,02(\mathrm{I})$ \\
\hline Paracetamol $^{\mathrm{b}}$ & $66 \pm 23,6$ & $24 \pm 3,1$ (III) & $0,07 \pm 0,02(\mathrm{I}, \mathrm{II}, \mathrm{III})$ \\
\hline $\mathrm{FHP}^{\mathrm{c}}$ & $61 \pm 20,4$ & $25 \pm 7,6$ & $0,06 \pm 0,02(I, I I)$ \\
\hline Perejil $^{\mathrm{d}}$ & $45 \pm 19,4$ & $21 \pm 5,3$ (III) & $0,05 \pm 0,01(\mathrm{I}, \mathrm{III})$ \\
\hline
\end{tabular}

${ }^{a}$ Sin tratamiento. ${ }^{b}$ Tratamiento: paracetamol

${ }^{c}$ Tratamiento: paracetamol y Purinor ${ }^{\circledR}$ (FHP)

${ }^{d}$ Tratamiento: paracetamol y Petroselinum sativum (perejil)

${ }^{e}$ Media $\pm S D ; N S:$ No significativo

${ }^{*} p<0,05$ : Test Kruskal-Wallis = I (entre todos los grupos), Test Mann-Whitney = II (paracetamol/FHP), III (paracetamol/perejil)

${ }^{f}$ AST: Aspartato aminotransferasa

${ }^{g}$ ALT: Alanina aminotransferasa

${ }^{h}$ GGT: $\gamma$-glutamil transferasa
Por otro lado, los niveles séricos de la gamma glutamil transferasa presentaron diferencia estadísticamente significativa entre todos los grupos, $p<0,001$ ( $p=0,000$, prueba Kruskal-Wallis). También, se pudo observar que al comparar dos grupos sí hubo diferencia estadísticamente significativa para la prueba de Mann-Whitney, $p<0,05$, en el grupo de paracetamol con FHP $(p=0,038)$, y $p<0,005$ en el caso de paracetamol con perejil $(p=0,003)$ (Tabla 2).

En relación con la concentración de las proteínas totales séricas, no hubo diferencia estadísticamente significativa a un $p<0,05$, mostrando los mayores niveles el grupo con tratamiento a base de perejil $(4,92 \mathrm{~g} / \mathrm{dL})$ y los menores niveles el grupo sin tratamiento o control $(4,76 \mathrm{~g} / \mathrm{dL})$ (Tabla 3$)$. Con respecto a la concentración de albúmina, tampoco hubo diferencia estadísticamente significativa a un $p<0,05$, pudiéndose observar mayores niveles en el grupo de paracetamol $(3,32 \mathrm{~g} / \mathrm{dL})$ y menores niveles en el grupo de perejil (3,10 g/dL) (Tabla 3$)$.

Tabla 3. Proteínas totales, albúmina y grupos sulfidrilos en suero sanguíneo de ratas según tratamiento.

\begin{tabular}{lccc}
\hline Tratamiento & $\begin{array}{c}\text { Proteínas } \\
\text { totales }^{(\mathrm{NS})} \\
\mathrm{g} / \mathrm{dL}\end{array}$ & Albúmina $^{(\mathrm{NS})}$ & $\begin{array}{c}\text { Grupos } \\
\text { sulfhidrilos }^{(\mathrm{NS})} \\
\mathrm{mM}\end{array}$ \\
\hline Control $^{\mathrm{a}}$ & $4,76 \pm 0,94$ & $3,21 \pm 0,62$ & $0,58 \pm 0,36$ \\
Paracetamol $^{\mathrm{b}}$ & $4,77 \pm 0,96$ & $3,32 \pm 0,30$ & $0,67 \pm 0,41$ \\
FHP $^{\mathrm{c}}$ & $4,90 \pm 0,79$ & $3,16 \pm 0,29$ & $0,59 \pm 0,33$ \\
Perejil $^{\mathrm{d}}$ & $4,92 \pm 0,43$ & $3,10 \pm 0,52$ & $0,54 \pm 0,42$ \\
\hline
\end{tabular}

a Sin tratamiento. $b$ Tratamiento: paracetamol

${ }^{c}$ Tratamiento: paracetamol y Purinor ${ }^{\circledR}$ (FHP)

${ }^{d}$ Tratamiento: paracetamol y Petroselinum sativum (perejil)

${ }^{e}$ Media \pm SD; NS: No significativo

Observando los resultados sobre la concentración de los grupos sulfidrilos en suero sanguíneo y comparando todos los grupos al mismo tiempo, no hubo diferencia estadísticamente significativa a $p<0,05$, presentando los mayores valores el grupo con tratamiento de paracetamol $(0,67 \mathrm{mM})$ y los menores valores correspondieron al grupo con tratamiento de perejil $(0,54 \mathrm{mM})$, seguido del grupo control $(0,58 \mathrm{mM})$ (Tabla 3$)$; mientras que, con respecto a los grupos sulfidrilos del citosol hepático, donde tampoco hubo diferencia estadísticamente significativa entre todos los grupos a $p<0,05$. Las mayores concentraciones fueron 
Tabla 4. Proteínas, grupos sulfidrilos y TBARS en citosol hepático, fracción posmitocondrial, de ratas según tratamiento.

\begin{tabular}{lccc}
\hline Tratamiento & $\begin{array}{c}\text { Proteínas }^{(\mathrm{NS})} \\
\mathrm{g} / \mathrm{dL}\end{array}$ & $\begin{array}{c}\text { Grupos sulfhidrilos } \\
(\mu \mathrm{M} / \mathrm{mS})\end{array}$ & $\begin{array}{c}\text { TBARS prot.) }^{\mathrm{f}} 1^{-3} \\
\mu \mathrm{M} / \mathrm{g} \text { h.f. }\end{array}$ \\
\hline Control $^{\mathrm{a}}$ & $2,34 \pm 1,23$ & $72,9 \pm 27,3$ & $0,036 \pm 0,005(\mathrm{l})$ \\
Paracetamol $^{\mathrm{b}}$ & $2,22 \pm 1,31$ & $85,8 \pm 42,8$ & $0,038 \pm 0,006(\mathrm{l}, \mathrm{Il})$ \\
FHP $^{\mathrm{c}}$ & $2,27 \pm 1,28$ & $73,5 \pm 30,3$ & $0,040 \pm 0,008(\mathrm{l})$ \\
Perejil $^{\mathrm{d}}$ & $2,05 \pm 0,93$ & $70,4 \pm 21,2$ & $0,031 \pm 0,006(\mathrm{l}, \mathrm{Il})$ \\
\hline
\end{tabular}

${ }^{a}$ Sin tratamiento. ${ }^{b}$ Tratamiento: paracetamol

${ }^{c}$ Tratamiento: paracetamol y Purinor ${ }^{\circledR}$ (FHP)

${ }^{d}$ Tratamiento: paracetamol y Petroselinum sativum (perejil).

${ }^{e}$ Media $\pm S D ; N S:$ No significativo

$* p$ <0,05: Test Kruskal-Wallis = I (Entre todos los grupos), Test Mann-Whitney = II (Paracetamol/perejil)

${ }^{f}$ TBARS: Especies reactivas al ácido tiobarbitúrico

encontradas en el grupo de paracetamol $[(85,8$ $\mu \mathrm{M} / \mathrm{mg}$ proteína) $\times 10^{-3}$ ], y los menores valores fueron para el grupo del perejil $[(70,4 \mu \mathrm{M} / \mathrm{mg}$ proteína) x $10^{-3}$ ] (Tabla 4 ).

La determinación de las especies reactivas al ácido tiobarbitúrico (TBARS) o radicales libres del citosol hepático permite en este trabajo mostrar que existe diferencia estadísticamente significativa a un $p<0,001$ entre todos los grupos $(p=0,000$, prueba Kruskal-Wallis). También, se pudo observar que al comparar dos grupos sí hubo diferencia estadísticamente significativa para la prueba de Mann-Whitney, a un $p<0,01$, en el caso de paracetamol con perejil $(p=0,008)$ (Tabla 4$)$.

Tabla 5. G-6-P deshidrogenasa, superóxido dismutasa y catalasa ${ }^{e}$ en citosol hepático, fracción posmitocondrial, de ratas según tratamiento.

\begin{tabular}{lccc}
\hline Tratamiento & $\begin{array}{c}\text { G-6-P DH } \\
(\mu \mathrm{M} / \mathrm{min} / \\
\text { mg prot.)x10 }\end{array}$ & $\begin{array}{c}\text { Superóxido dismutasa }^{-3} \\
\mathrm{US} / \mathrm{min} / \\
\mathrm{mg} \text { prot. }\end{array}$ & $\begin{array}{c}\text { Catalasa(NS) }^{(\mathrm{NM})} \\
\mathrm{mg} / \mathrm{min} /\end{array}$ \\
\hline Control $^{\mathrm{a}}$ & $5,2 \pm 2,0(\mathrm{I})$ & $1,27 \pm 0,6$ & $28,07 \pm 16,0$ \\
PAracetamol $^{\mathrm{b}}$ & $5,7 \pm 2,6(\mathrm{I})$ & $1,73 \pm 1,0$ & $31,82 \pm 18,2$ \\
FHP $^{\mathrm{c}}$ & $7,8 \pm 4,7(\mathrm{I})$ & $1,37 \pm 0,6$ & $29,93 \pm 14,4$ \\
Perejil $^{\mathrm{d}}$ & $4,0 \pm 1,6(\mathrm{I})$ & $2,48 \pm 1,9$ & $28,46 \pm 12,4$ \\
\hline
\end{tabular}

${ }^{a}$ Sin tratamiento. ${ }^{b}$ Tratamiento: paracetamol

${ }^{c}$ Tratamiento: paracetamol y Purinor ${ }^{\circledR}$ (FHP)

${ }^{d}$ Tratamiento: paracetamol y Petroselinum sativum (perejil)

${ }^{e}$ Media $\pm S D ; N S:$ No significativo

${ }^{*} p<0,05$ : Test Kruskal-Wallis = I (Entre todos los grupos)
Así mismo, en los valores correspondientes a la actividad de la glucosa-6-fosfato deshidrogenasa (G-6-P DH) del citosol hepático, sí existe diferencia estadísticamente significativa a un $p<0,05$, entre todos los grupos ( $p=0,01$, prueba Kruskal-Wallis). También, se pudo observar que al comparar dos grupos sí hubo diferencia estadísticamente significativa para la prueba de Mann-Whitney, a un $p<0,10$, entre paracetamol con perejil $(p=0,06)$ (Tabla 5).

Con respecto a la actividad de la superóxido dismutasa, no hubo diferencia estadísticamente significativa entre todos los grupos a un $p<0,05$, pero, se pudo observar que la administración oral de perejil $(2,48 \mathrm{U} / \mathrm{min} / \mathrm{mg}$ proteína) produjo mayores valores, comparados con el grupo con tratamiento de paracetamol $(1,73 \mathrm{U} / \mathrm{min} / \mathrm{mg}$ proteína) y el grupo control (1,27 U/min/mg proteína) (Tabla 5). Así mismo, en lo referente a la actividad de la catalasa, no hubo diferencia estadísticamente significativa entre todos los grupos a un $p<0,05$, pero, se pudo observar que la administración oral de paracetamol $(31,82$ $\mu \mathrm{M} / \mathrm{min} / \mathrm{mg}$ proteína) produjo los mayores valores seguido del grupo con FHP $(29,93 \mu \mathrm{M} / \mathrm{min} / \mathrm{mg}$ proteína), presentando los menores valores el grupo con tratamiento de perejil $(28,46 \mu \mathrm{M} / \mathrm{min} / \mathrm{mg}$ proteína) y el grupo control $(28,07 \mu \mathrm{M} / \mathrm{min} / \mathrm{mg}$ proteína) (Tabla 5).

Macroscópicamente, se observó una ligera palidez superficial en los hígados de ratas tratadas solo con paracetamol. Los hígados de los otros grupos de ratas permanecieron sin alteraciones.

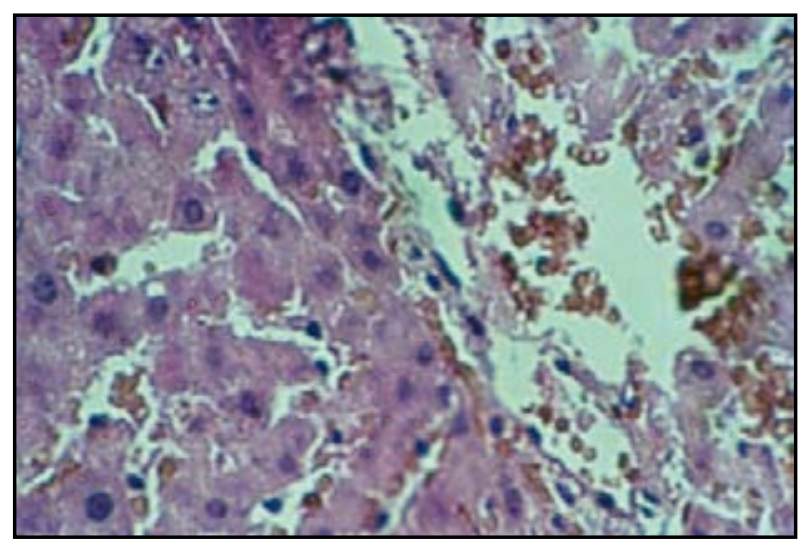

Figura 1. Hígado de rata sin tratamiento. No existen signos de necrosis. Los hepatocitos se encuentran preservados. Coloración: hematoxilina eosina, $(40 \mathrm{X})$. 
(a)

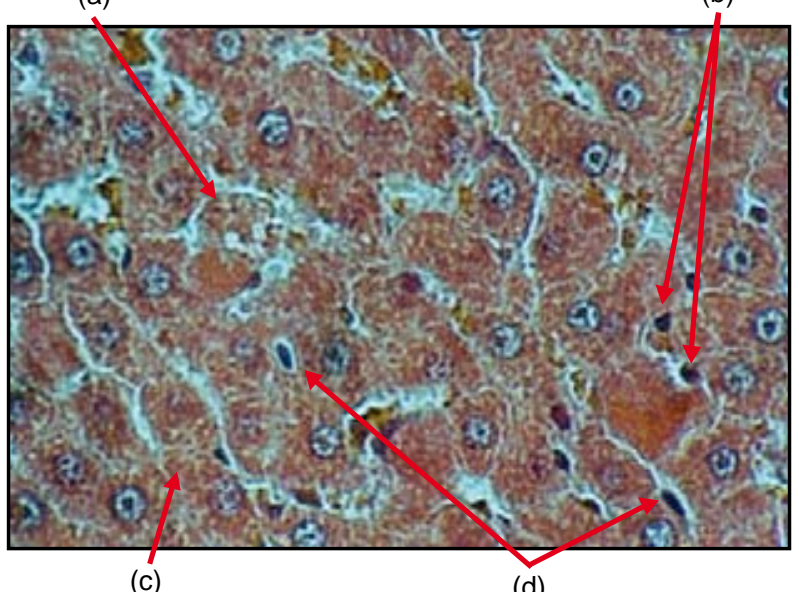

(c)

(d)

Figura 2. Hígado de rata con tratamiento: paracetamol. (a) Necrosis severa, (b) apreciándose restos nucleares.

(c) Retención biliar. (d) Hipertrofia de las células de Kupffer. Coloración: hematoxilina eosina, $(40 \mathrm{X})$.

Los hígados de ratas control (sin tratamiento) no presentaron alteraciones histológicas (Figura 1). En cambio, en las ratas que recibieron el tratamiento con paracetamol se observó signos de necrosis severa (Figura 2). Así mismo, los hígados de ratas tratadas con paracetamol y FHP mostraron signos (c)

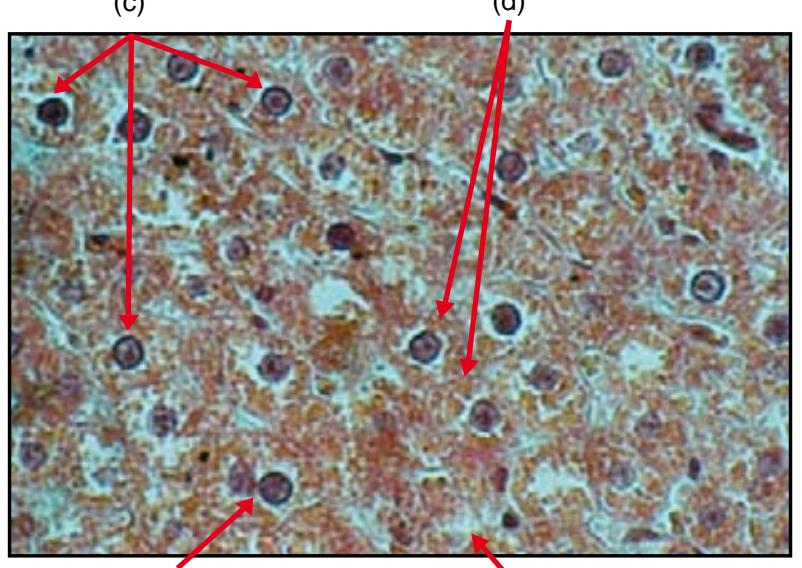

(b) (a)
Figura 3. Hígado de rata con tratamiento: paracetamol y Purinor ${ }^{\circledR}$ (FHP). (a) Necrosis severa con ausencia de membrana citoplasmática de hepatocitos y (b) homogenización de la cromatina nuclear, (c) mostrándose la membrana del núcleo de los hepatocitos ligeramente engrosada. (d) Se observa pigmento pardo amarillento localizado en el citoplasma.

Coloración: hematoxilina eosina, (40 X). de necrosis entre moderada y severa (Figura 3). Por otro lado, se pudo observar, histológicamente, en los hígados de ratas tratadas con paracetamol y perejil, que mayormente permanecían sin alteraciones o con signos de necrosis leve, presentando algunas de ellas signos de necrosis moderada acompañados de regeneración celular marcada (Figura 4). Se encontró diferencia histológica estadísticamente significativa entre los grupos, a un $p<0,05$.

\section{DISCUSIÓN}

La toxicidad hepática producida por la administración de paracetamol ha sido descrita por diversos autores. Existen estudios que muestran que el paracetamol se metaboliza a nivel hepático por varias vías, conduciendo una de ellas a la reacción de conjugación con el ácido glucurónico, mientras que la segunda vía se conjuga con el sulfato, reacciones que tornan inocuo al paracetamol. En cambio, existe otra reacción que lo conduce a la formación de $\mathrm{N}$-acetil imidoquinona, compuesto que tiene la propiedad de realizar un ataque nucleofílico a diversos componentes celulares. Los compuestos dadores de grupos sulfidrilo, como el glutatión, ejercen un eficiente efecto protector hepático, por (b)

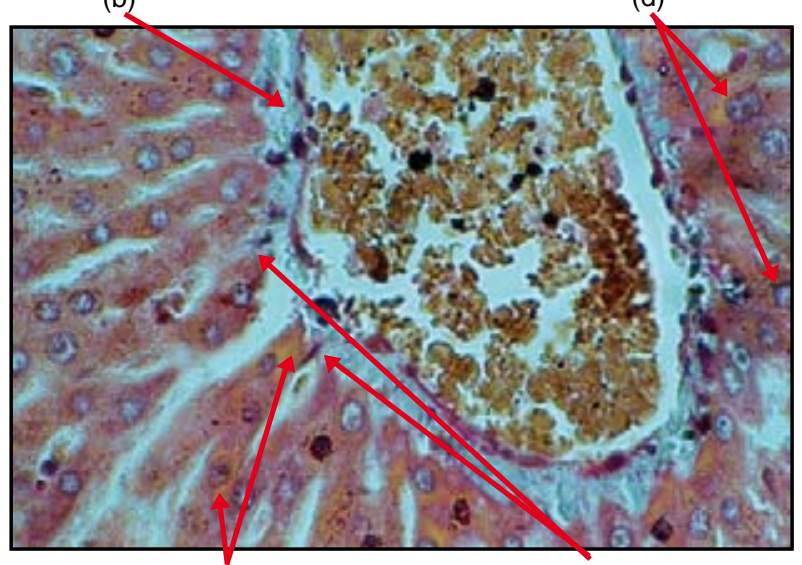

(a) (c)
Figura 4. Hígado de rata con tratamiento: paracetamol y Petroselinum sativum (perejil). (a) Necrosis moderada con retención de pigmento pardo amarillento. (b) Espacio portal normal. (c) Células de Kupffer normales. (d) Signos de regeneración por presencia de hepatocitos binucleados. Coloración: hematoxilina eosina, $(40 \mathrm{X})$. 
cuyo motivo la administración de $\mathrm{N}$-acetil cisteína por vía endovenosa permite evitar el efecto nocivo que ejercen los radicales libres generados por los metabolitos del paracetamol $\left({ }^{34}\right)$.

El paracetamol es un medicamento que, administrado a elevadas dosis, causa daño hepático. Este proceso se realiza por generación de radicales libres. En tal sentido, la administración de sustancias con propiedades antioxidantes podría evitar o disminuir el efecto tóxico de la mencionada droga. El daño a nivel hepático puede ser evaluado de diversas formas; una de ellas lo constituye la medición de la actividad sérica de enzimas como GPT, GOT o GGT, cuya elevación en el plasma pondría en evidencia el daño celular que causa el paracetamol.

En nuestro estudio, el incremento del nivel sérico de transaminasas que observamos en aquellas ratas que recibieron el tratamiento con paracetamol constituye una prueba del daño hepático que ha ejercido este medicamento. Dicho efecto se corrobora con el estudio histopatológico, que permite mostrar signos de necrosis hepatocelular severa. Se ha descrito, en otros trabajos, que una sobredosis de paracetamol causa una necrosis hepática aguda $\left.{ }^{(35}\right)$, ocasionada, probablemente, por productos derivados del metabolismo del paracetamol $\left({ }^{36}\right)$. Además, el grupo de ratas al que se le administró FHP, compuesto que tiene propiedades hepatoprotectoras, mostró mayormente necrosis entre moderada y severa, presentando, así mismo, los mayores valores en AST, ALT y GGT, lo que nos indicaría muy poca o ninguna protección hepática contra el daño inducido por paracetamol, que genera radicales libres en su mecanismo de acción. En cambio, las ratas tratadas con perejil no mostraron mayores alteraciones tisulares. Esta observación mantiene correlación con los niveles de transaminasas, principalmente con GGT y ALT, donde hay diferencia estadísticamente significativa, que fueron los más bajos, lo que indicaría que este alimento ejerció un mejor efecto hepatoprotector.

En un estudio realizado con ratas tornadas diabéticas pudo observarse cambios degenerativos en sus hepatocitos, modificaciones que fueron reducidas significativamente en las ratas que recibieron tratamiento con Petroselinum crispum. Así mismo, las ratas diabéticas mostraron elevados niveles de ALT y fosfatasa alcalina. En cambio, el grupo de ratas a las que se les administró Petroselinum crispum tuvo niveles significativamente más bajos de las enzimas antes citadas $\left({ }^{37}\right)$.

La vía de las pentosas tiene como principal función proporcionar ribosa-5-fosfato necesaria para la síntesis de novo de ácidos nucleicos y compuestos reductores (NADPH) requeridos en las reacciones biosintéticas; estos procesos son importantes en la mucosa intestinal, glándula mamaria y tejido adiposo. La glucosa-6-fosfato deshidrogenasa es una enzima que participa en la vía de las pentosas encargada de generar NADPH, por cuyo motivo, su determinación es importante, especialmente en el hígado, a causa de la función que cumple este órgano frente a la acción de agentes hepatotóxicos, para cuyo propósito, se requiere apreciables cantidades de NADPH y ribosa-5-fosfato para los procesos de activación y desintoxicación, así como, para la síntesis y reparación de ácidos nucleicos.

Se ha descrito que la administración de tioacetamida a ratas durante un periodo de 60 días ocasiona un incremento de la actividad de glucosa-6-fosfato deshidrogenasa a las 4 semanas de tratamiento, no habiéndose observado un incremento perceptible de dicha actividad en la primera semana de aplicación del hepatotóxico, resultado, que es, en cierto modo, similar al que hemos encontrado $\left({ }^{38}\right)$. En este trabajo, el incremento que se observa en la actividad de la glucosa-6-fosfato deshidrogenasa se produce como consecuencia de la necesidad que tiene la célula de generar compuestos reductores (NADPH), para protegerse de la acción tóxica del paracetamol. En cambio, el grupo de ratas que recibió el tratamiento con perejil no presentó incremento significativo alguno. Mientras que, el grupo de ratas que recibió el tratamiento con el hepatoprotector (FHP), mostró un incremento de la actividad de esta enzima, inclusive mayor que el grupo de ratas que solo recibió el paracetamol, efecto que guarda relación con el incremento de TBARS que se observa en los grupos de ratas que recibieron los tratamientos solo con paracetamol y con paracetamol y el FHP. En cambio, se observa una disminución de TBARS en los grupos que se les administró paracetamol y perejil, resultado que evidencia el efecto antioxidante de este alimento. 
Normalmente, se puede ingerir con la dieta sustancias naturales con capacidad antioxidante, como flavonoides del tipo de isoflavonas (genisteína, daidzeína), myricetina, quercetina; polifenoles, taninos, $\beta$-caroteno, vitamina $E$, vitamina $C$, entre otros $\left({ }^{2,10}\right)$. Es así como, un equipo de investigadores estudió la capacidad antioxidante de los extractos metanólicos de frutos, corteza y hojas de laurel (Laurus nobilis) frente al sistema $\mathrm{Fe}^{2+}$ /ascorbato midiendo TBARS y encontraron que la corteza presentaba mucha mayor capacidad antioxidante $\left({ }^{39}\right)$.

Como se ha sugerido, la administración de paracetamol ejerce un efecto dañino a nivel hepático mediante la formación de radicales libres los cuales tienen la propiedad de oxidar a los lípidos de las membranas plasmáticas y como consecuencia de ello se forman sustancias, como el malondialdehído, que reaccionan con el ácido tiobarbitúrico, por cuyo motivo, la determinación de TBARS en el homogenizado de hígado constituye una de las maneras de evaluar el efecto nocivo ejercido por el Paracetamol.

En el presente trabajo, la generación de especies reactivas al tiobarbitúrico (TBARS) muestra diferencias estadísticamente significativas en todos los grupos experimentales, correspondiendo el valor más bajo para los grupos que recibieron el tratamiento con perejil, resultado que podría indicar una acción antioxidante, aunque algo moderada. Los niveles de la superóxido dismutasa, enzima que participa en la transformación del radical anión superóxido, en citosol hepático, fracción posmitocondrial, fue mayor en todos los grupos experimentales con relación al grupo control, ya sea que hubiesen recibido el tratamiento solo con paracetamol o, más aún, con esta droga y perejil o FHP. Además, la actividad de la catalasa, enzima que participa en las reacciones de transformación del peróxido de hidrógeno, fue mayor en el grupo que recibió solamente paracetamol, mientras que, en los grupos restantes, incluido el grupo control, la actividad de esta enzima estuvo más baja. Mientras tanto, en otros estudios, la administración de una dosis única de tioacetamida, compuesto que también produce daño hepático a través de la generación de radicales libres, ocasiona una disminución de las actividades de superóxido dismutasa y catalasa en hígado de rata, siendo más notable esta caída de la actividad a las 48 horas de haberse administrado el hepatotóxico $\left({ }^{40}\right)$.

Al estudiar los investigadores en un grupo de ratas el efecto hepatotóxico del tetracloruro de carbono, se observó una disminución en la albúmina sérica y proteínas totales acompañadas de un incremento en AST, ALT y fosfatasa alcalina; también, hubo alteraciones hepáticas, como esteatosis, degeneración hidrópica y necrosis. $\mathrm{Al}$ mismo tiempo, administraron a otro grupo de ratas, $\mathrm{CCl}_{4}$ y un extracto de Centella asiática (L) y observaron una acción hepatoprotectora, que fue atribuida a la presencia de asiaticósido $(14,5 \%)$ en el extracto $\left({ }^{41}\right)$. Otros estudios comprobaron en ratones el efecto antioxidante y antiinflamatorio del extracto etanólico de las flores de la Tabernaemontana coronaria $(\mathrm{L}) \mathrm{R}$. $\mathrm{Br}\left({ }^{42}\right)$. Así mismo, otros investigadores estudiaron en ratas y ratones la actividad antioxidante del extracto etanólico de Tephrosia purpurea Linn frente al $\mathrm{CCl}_{4} \mathrm{y}$ pudieron mostrar una significativa inhibición de la lipoperoxidación y de la generación de radical anión superóxido $\left({ }^{43}\right)$.

En otro estudio, se comunicó que el Rosmarinus officinalis (Lamiaceae) puede aliviar la hepatotoxicidad aguda inducida por el $\mathrm{CCl}_{4}$ en ratas, posiblemente evitando la formación de radicales libres generados durante el metabolismo del $\mathrm{CCl}_{4}$. Su principio activo sería el carnosol, uno de los principales constituyentes del Rosmarinus officinalis (Lamiaceae) que ha mostrado actividad antioxidante $\left({ }^{44}\right)$.

En relación al contenido de grupos sulfidrilos en citosol de hígado de rata, fracción posmitocondrial, observamos, en el presente estudio, que es mayor en el grupo que recibió solo paracetamol que en el grupo control, y el grupo que recibió, además de paracetamol, perejil, mostró valores menores, aún que el grupo control, lo que sugiere que el tratamiento con este alimento inhibió el incremento en el contenido de los grupos sulfidrilos ocasionado probablemente por el paracetamol y otras noxas.

Un hecho similar ocurre en el suero, donde es posible apreciar que los niveles de grupos sulfidrilos son mayores en el grupo con paracetamol que en los 
diferentes grupos experimentales y el control, lo que sugeriría que el paracetamol podría ser el causante de este incremento. Otros autores describen que, después de la administración de tioacetamida, se observa una disminución de niveles intracelulares de grupos sulfidrilos de las proteínas de hepatocitos de ratas a las 24 y 48 horas postratamiento $\left({ }^{40}\right)$.

Finalmente, el estudio histopatológico realizado pudo corroborar que los hígados de las ratas que recibieron solamente paracetamol fueron los que presentaron mayores signos de necrosis severa, es decir, fueron más dañados. Así mismo, el grupo que recibió, además de paracetamol, el FHP, mostró signos de necrosis entre moderada y severa, lo que indicaría una mínima hepatoprotección. Estos daños hubieran sido mayores aún si las ratas hubieran presentado signos de desnutrición aguda, la cual hubiera afectado su metabolismo, el volumen de distribución y la depuración (clearance) de una dosis terapéutica que harían más severas las lesiones $\left({ }^{45}\right)$.

En cambio, el grupo que recibió paracetamol y perejil mostró algunos signos de necrosis hepática leve o ausencia de ellos y, además, signos de regeneración parenquimal, con lo cual se estaría evidenciando el gran efecto hepatoprotector de los componentes de este alimento.

En los estudios de otros investigadores se pudo observar que la sustancia natural PFL presenta actividad antioxidante tanto in vitro como in vivo y protege contra la hepatotoxicidad inducida por $\mathrm{CCl}_{4}$, en dos semanas de tratamiento, evaluado mediante valores de AST, ALT, examen histológico y la medición de malondialdehído, producto de lipoperoxidación $\left({ }^{46}\right)$. Así mismo, otros investigadores estudiaron la sustancia de origen vegetal LIV-52, la cual mostró efecto hepatoprotector evaluado por transaminasas, al igual que por estudios histopatológicos en los animales tratados $\left({ }^{47}\right)$.

Podemos concluir, de todo lo observado en el presente estudio de investigación, que el perejil ejerce un efecto antioxidante y hepatoprotector efectivo a nivel hepático en ratas con intoxicación inducida por paracetamol, evidenciado a través de ALT, GGT, glucosa-6-fosfato deshidrogenasa, TBARS y observaciones histopatológicas.

\section{REFERENCIAS BIBLIOGRÁFICAS}

1. Martín-Sanz P, Cascales M. Metabolismo de fármacos en hígado y sus alteraciones. En: Cascales M y col. Hepatología. Madrid: Consejo Superior de Investigaciones Científicas; 1990.

2. Yu BP. Cellular defenses against damage from reactive oxygen species. Physiologycal Reviews. 1994;74(1):139-62.

3. Cheeseman K, Slater T. An introduction to free radicals in medicine. Brit Med Bull. 1993;49:481-93.

4. Padh H. Vitamin C: Newer insights into its biochemical functions. Nutr Rev. 1991;49(3):65-70.

5. Troncoso L. Efecto del ascorbato sobre la ruptura de los puentes disulfuro. Tesis para optar el Grado Académico de Magíster en Nutrición. Lima: Facultad de Medicina, Universidad Nacional Mayor de San Marcos; 1996.

6. Fraga C, Shigenaga M, Park J, Degan P, Ames B. Vitaminas antioxidantes: bioquímica, nutrición y participación en la prevención de ciertas patologías. BEB. 1995;14(1):12-7.

7. Guija E, Troncoso L. Radicales libres y envejecimiento. Bol Soc Quím Perú. 2000;66:33-51.

8. Edgington $\mathrm{S}$. As we live and breathe: Free radicals and aging. Bio Tech. 1994;12:37-40.

9. Singh V, Gaby S. Premaligmant lesions: role of antioxidant vitamins and $\beta$-carotene in risk reduction and prevention of maligmant transformation. Am J Clin Nutr. 1991;53:386s-90s.

10. Halliwell B, et al. How to characterize a biological antioxidant. Free Rad Res Comms. 1990;9(1):1-32.

11. Cañigueral S, Vanaclocha B. Usos terapéuticos del tomillo. Rev Fitot. 2000;1(1):5-13.

12. Liu F, Ng TB. Antioxidative and free radical scavenging activities of selected medicinal herbs. Life Sci. 2000;66(8): 725-35.

13. Troncoso L, Guija E. Propiedades antioxidantes del perejil (Petroselinum sativum). Rev Soc Quím Perú. 2005;71(2):99106.

14. Troncoso L, Guija E, Ronceros S, Valdivia F, Flores J, Guija H y col. Efecto del camu-camu (Myrciaria dubia) y perejil (Petroselinum sativum) en plasma sanguíneo de pacientes con dislipidemia y diabetes mellitus tipo 2. Libro de resúmenes. III Jornadas de Investigación en Salud. Lima: Facultad de Medicina, UNMSM; 2001.

15. Troncoso L, Nolberto V, Oliveira G, Torrealva L, Guija H, Quiroz K. Estudio fitoquímico del Petroselinum sativum (perejil): Separación y caracterización de fracciones con capacidad antioxidante. Libro de resúmenes. III Jornadas Científicas Sanfernandinas y VI Jornadas de Investigación en Salud. An Fac Med. 2004;65 Supl 1:S20-21.

16. Troncoso L, Guija E, Oliveira G, Palomino F, Norberto V, Quiroz K. Evaluación toxicológica aguda del extracto acuoso y fracción B (extracción con etanol y agua) del perejil (Petroselinum sativum) en ratas. Libro de resúmenes. IV Jornadas Científicas Sanfernandinas y VII Jornadas de Investigación en Salud. An Fac Med. 2005;66 Supl 1:S26.

17. Troncoso L, Guija E, Bernui I, Cairo Y, Oriondo R, Pacheco A. Capacidad antioxidante de alimentos y plantas medicinales. 
Libro de resúmenes. Primer Congreso Internacional FITO 2000 y 1er Congreso Peruano de Plantas Medicinales y Fitoterapia. Lima: Instituto de Fitoterapia Americano; 2000.

18. Troncoso L, Guija E, Quiroz K. Capacidad antioxidante del "Chimichurri" y sus componentes. Libro de resúmenes. Segundo Congreso Internacional FITO 2003 y 2do Congreso Peruano de Plantas Medicinales y Fitoterapia. Lima, Perú: Instituto de Fitoterapia Americano; 2003.

19. Troncoso L, Guija E, Quiroz K. Capacidad antioxidante de los condimentos del "Chimichurri". Libro de resúmenes. Segundo Congreso Internacional FITO 2003 y 2do Congreso Peruano de Plantas Medicinales y Fitoterapia. Lima, Perú: Instituto de Fitoterapia Americano; 2003.

20. Troncoso L, Guija E, Quiroz K. Efecto de la temperatura sobre la generación de radicales libres y la capacidad antioxidante del Capsicum annuum L. (Pimiento). Libro de resúmenes. Segundo Simposium Internacional de Plantas Medicinales y Fitoterapia. FITO 2004. Lima, Perú: Instituto de Fitoterapia Americano; 2004.

21. Troncoso L, Guija E, Quiroz K. Efecto de la temperatura sobre la capacidad antioxidante de la Allium cepa L. var. Aggregatum (Cebolla china). Libro de resúmenes. XXII Congreso Peruano de Química. Lima, Perú: Sociedad Química del Perú; 2004.

22. Troncoso L, Guija E, Quiroz K. Capacidad antioxidante de la Beta vulgaris L. var. Crassa (Betarraga). Libro de resúmenes. I Congreso Internacional y VII Congreso Peruano de Nutrición “Alimentación y Nutrición para una Vida Saludable”. Lima, Perú: Sociedad Peruana de Nutrición; 2004.

23. D'Arcy P. Adverse reactions and interactions with herbal medicines. Part II. Drug interactions. Adverse Drug React Toxicol Rev. 1993;12(3):147-62.

24. Hernández J, Valero H, Gil R. 23 especies vegetales medicinales de uso frecuente en la población de Tabay. Rev Fac Farma. 2002;44(2):51-8.

25. Kreydiyyeh S, Usta J. Diuretic effect and mechanism of action of parsley. J Ethnopharmacol. 2002;79:353-7.

26. Frankel S, Reitman S, Sonnenwirth AC, editors. Gradwohl's Clinical Laboratory Methods and Diagnosis. $7^{\text {a }}$ Ed. Vol. 1. Saint Louis: The C. V. Mosby Company; 1970.

27. Wahlefeld A, Bergmeyer, H. En: Bergmeyer H (Ed.). Methods of Enzymatic Analysis, Vol. III. $3^{\text {rd }}$ ed. Weinheim: Verlag Chemie; 1987.

28. Ellman G. Tissue sulphydryl groups. Arch Biochem Biophys. 1959;82:70-5.

29. Wiener lab Group. Vademecum. Reagents for Clinical laboratories. Rosario: Wiener lab Group; 2000.

30. Del Maestro R, McDonald W, Anderson R. Superoxide dismutase, catalase and glutathione peroxidase in experimental and human brain tumors. En: Greenwald R, Cohen G, editors. Oxygen Radicals and their Scavenyer Systems, Vol. 2. New York: Elsevier; 1983.

31. Aebi H. En: Bergmeyer H (Ed.). Methods of Enzymatic Analysis. Vol. III, $3^{\text {rd }}$ ed. Weinheim: Verlag Chemie; 1987.

32. Deutsch J. In: Bergmeyer H (Ed.). Methods of Enzymatic Analysis. Vol. III, $3^{\text {rd }}$ ed. Weinheim: Verlag Chemie; 1987.
33. Laudicina D, Marnett L. Enhancement of hydroperoxidedependent lipid peroxidation in rat liver microsomes by ascorbic acid. Arch Biochem Biophys. 1990;278:73-80.

34. Prescott L, Critchley J. Intravenous $\mathrm{N}$-acetilcisteína: the treatment of choice for paracetamol poisoning. Brit Med J. 1979;2:1097-100.

35. Davidson D, Eastham W. Acute liver necrosis following overdosage of paracetamol. Brit Med J. 1966;2:497-9.

36. Davies M, Labadarios D, Williams R. Metabolism of paracetamol and after therapeutic hepatotoxic doses in man. J Inter Med Res. 1976;4:40-5.

37. Bolkent S, Yanardag R, Ozsoy-Sacan O, Karabulut-Bulan O. Effects of parsley (Petroselinum crispum) on the liver of diabetic rats: a morphological and biochemical study. Phytother Res. 2004;18(12):996-9.

38. Martín-Sanz P, Cascales C, Cascales M. Lipogenesis and cholesterogenesis de novo in liver and adipose tissue. Alterations of lipid metabolism by the effect of short- and long-term thioacetamide administration to rats. Carcinog. 1989;10(3):477-81.

39. Simic M, Kundakovic T, Kovacevic N. Preliminary assay on the antioxidative activity of Laurus nobilis extracts. Fitot. 2003;74(6):613-6.

40. Sanz N, Diez-Fernandez C, Fernandez-Simon L, Alvarez A, Cascales, M. Necrogenic and regenerative responses of liver of newly weaned rats against a sublethal dose of thioacetamide. Biochem Biophys Acta. 1998;1384:66-78.

41. Antony B, Santhakumari G, Merina B, Sheeba V, Mukkadan J. Hepatoprotective effect of Centella asiática (L) in carbon tetrachloride-induced liver injury in rats. Indian J Pharm Sci. 2006;68(6):772-6.

42. Thambi P, Kuzhivelil B, Sabu M, Jolly C. Antioxidant and antiinflammatory activities of the flowers of Tabernaemontana coronaria (L) R. Br. Indian J Pharm Sci. 2006;68(4):456-60.

43. Soni K, Suresh Kumar P, Saraf M. Antioxidant activity of fraction of Tephrosia purpurea Linn. Indian J Pharm Sci. 2006;68(3):352-5.

44. Sotelo-Felix J, Martinez-Fong D, Muriel P. Protective effect of carnosol on $\mathrm{CCl}_{4}$-induced acute liver damage in rats. Europ J Gastr Hepatol. 2002;14(9):1001-6.

45. González M, Vicuña N, Naranjo R, Mora J, Marquez J, García G. Efectos de la desnutrición aguda sobre el metabolismo del acetaminofén. Rev Fac Farma. 2002;44(2):7-13.

46. Pérez J, Sánchez N, Bu M. Actividad antioxidante in vivo e in vitro de un extracto natural de origen vegetal. Rev Cubana Plant Med. 1998;3(3):19-22.

47. Shivani P. Hepatoprotective effect of LIV-52 against $\mathrm{CCl}_{4}$ induced lipid peroxidation in liver of rats. Indian J Exp Biol. 1984;32:674-5.

Manuscrito recibido el 17 de octubre de 2007 y aceptado para publicación el 13 de diciembre de 2007.

Correspondencia: Dra. Luzmila Victoria Troncoso Corzo

Centro de Investigación de Bioquímica y Nutrición.

Facultad de Medicina, UNMSM.

Av. Grau 755. Lima 1, Perú

Correo-e: 1troncoso@terra.com.pe 